\title{
Synthesis of Some Benzimidazole Derivatives Bearing 1,3,4-Oxadiazole Moiety as Anticancer Agents
}

\author{
BEREKET MOCHONA $^{1^{*}}$, ELIZABETH MAZZIO ${ }^{2}$, MADHAVEI GANGAPURUM ${ }^{2}$, \\ NELLY MATEEVA ${ }^{1}$ and K. K. REDDA ${ }^{2}$
}

${ }^{1}$ College of Science and Technology, Department of Chemistry, ${ }^{2}$ College of Pharmacy and Pharmaceutical Sciences, Florida A\&M University, Tallahassee, FL 32307, USA

bereket.mochona@famu.edu

Received 11 February 2015 / Accepted 4 March 2015

\begin{abstract}
In an effort to establish new benzimidazole related structural leads with improved anticancer activity, several new benzimidazole derivatives (5a-i) with 1,3,4-oxadiazole scaffold incorporated were synthesized and studied for their anticancer activity. The anticancer screening against MDA-MB-231 breast cancer cell lines showed that compound (5c) exhibited moderate cytotoxicity.
\end{abstract}

Keywords: Benzimidazole, 1,3,4-Oxadizole, Pharmacophore hybridization, Cytotoxcity

\section{Introduction}

Benzimidazole derivatives are structural isosters of naturally occurring nucleotides, thus they can interact with biological macromolecules such as proteins, enzymes and receptors. The unique structural features and a wide range of biological activities of benzimidazole made it privileged structure in drug discovery ${ }^{1-4}$. Apart from benzimidazoles, the five membered azole heterocycles, especially, oxadiazole has been introduced into drug discovery for several purposes. Molecular modeling and pharmacokinetic studies have demonstrated that incorporating oxadiazole moiety to drug-like molecules change their polarity, flexibility as well as metabolic profile and ability to engage in hydrogen bonding. Hence, oxadiazoles have been widely employed as bioesteric replacement of carbonyl compounds, such as carbamates, hydroxamic esters, amides and esters in a number of biological targets ${ }^{5-9}$.

The above mentioned considerations prompted us to design a new series of benzimidazole derivatives with 1,3,4-oxadiazole moieties incorporated into benzimidazole nuclei. Here in, we report the synthesis (Scheme 1), characterization and cytotoxic screening of a new class of 1, 3, 4-oxadazolylbenzimidazole. 


\section{Experimental}

Reagents and solvents were purchased from Sigma-Aldrich Chemical Company Inc. and used as received. Melting points were determined in open capillaries on a Gallenkamp digital melting point apparatus and were uncorrected. The Infrared spectra were recorded in KBr discs using Shimadzu FT-IR 8000 spectrometer. ${ }^{1} \mathrm{H}$ NMR (DMSO- $\mathrm{d}_{6}$ ) and ${ }^{13} \mathrm{C}$ NMR spectra were recorded using Bruker $300 \mathrm{MHz}$ spectrometer using TMS as internal standard. Thin-layer chromatography was performed using precoated silica gel plates (silica gel $\left.0.25 \mathrm{~mm}, 60 \mathrm{G} \mathrm{F}_{254}\right)$.<smiles>[R]c1ccc(C(=O)OCC)cc1</smiles><smiles>[R]c1ccc(-c2nnc(SCc3nc4ccccc4[nH]3)o2)cc1Cl</smiles>

$$
\begin{array}{llll}
\mathbf{5 a} & \mathrm{R}=\mathrm{H} & \mathbf{5 f} & \mathrm{R}=\mathrm{Cl} \\
\mathbf{5 b} & \mathrm{R}=\mathrm{OCH}_{3} & \mathbf{5 g} & \mathrm{R}=-\mathrm{C}\left(\mathrm{CH}_{3}\right)_{3} \\
\mathbf{5 c} & \mathrm{R}=\mathrm{F} & \mathbf{5 h} & \mathrm{R}=\mathrm{NH}_{2} \\
\mathbf{5 d} & \mathrm{R}=\mathrm{NO}_{2} & \mathbf{5 i} & \mathrm{R}=\mathrm{Br} \\
\mathbf{5 e} & \mathrm{R}=\mathrm{CH}_{3} & &
\end{array}
$$<smiles>[R]c1ccc(-c2nnc(SCc3nc4ccccc4n3Cc3ccccc3)o2)cc1</smiles>

Scheme 1. Reagents and condition (a) $\mathrm{N}_{2} \mathrm{H}_{4} \mathrm{H}_{2} \mathrm{O}$, Ethanol, Reflux; (b) $\mathrm{CS}_{2}, \mathrm{KOH}$, Ethanol; (c) $\mathrm{K}_{2} \mathrm{CO}_{3}$, D-glucose in DMF or NaOAc, Ethanol, $\mathrm{XCH}_{2} \mathrm{Ph}$; (d) $\mathrm{NaH}$,

\section{General procedure for compounds $\mathbf{3 a}-\mathbf{i}$}

Synthesized by a method reported in literature ${ }^{10}$. To a solution of substituted benzohydrazide (2a-i) $(0.01 \mathrm{~mol})$ in ethanol $(25 \mathrm{~mL})$ at $0{ }^{\circ} \mathrm{C}$, carbon disulphide $(2 \mathrm{~mL})$ and potassium hydroxide $(0.6 \mathrm{~g})$ were added and the reaction mixture was refluxed until the evolution of $\mathrm{H}_{2} \mathrm{~S}$ gas ceased (3- $4 \mathrm{~h}$ ). Excess solvents were evaporated under reduced pressure and the residue was dissolved in water and then acidified with dilute hydrochloric acid (10\%) to pH 5. The precipitate was filtered off, dried and crystallized from ethanol.

\section{5-(4-Methoxyphenyl)-1,3,4-oxadiazole-2-thiol (3b)}

White solid,Yield: 76\%; mp: $169-171{ }^{\circ} \mathrm{C} ;{ }^{1} \mathrm{H}$ NMR (300 $\left.\mathrm{MHz}, \mathrm{CDCl}_{3}\right) \quad \delta: 3.75(\mathrm{~s}, 3 \mathrm{H})$, 7.08-7.21 (m, 3H, Ar- H), 7.59-7.64 (m, 1H, Ar-H), 7.70 (d, 1H, J = 7.0, Ar-H), 7.82 (d, $1 \mathrm{H}, \mathrm{J}=7.0, \mathrm{Ar}-\mathrm{H}), 7.95(\mathrm{~s}, 1 \mathrm{H}, \mathrm{Ar}-\mathrm{H})$.

General procedure for the synthesis of $\mathbf{4 a - i}$

A mixture of $3(0.01 \mathrm{~mol}), \mathrm{K}_{2} \mathrm{CO}_{3}(0.012 \mathrm{~mol}), D$-glucose $(0.004 \mathrm{~mol}), 2$-chloromethylbenzimidazole $(0.01 \mathrm{~mol})$ and DMF $(35 \mathrm{~mL})$ was stirred at room temperature for $2 \mathrm{~h}$. At the end of this period, the reaction mixture was poured into ice-water. The separated product was filtered, washed with water, dried and recrystallized from ethyl acetate to obtain pure 4 .

\section{2-(5-Phenyl-1,3,4-oxadiazol-2-ylthio)methyl)-1H-benzimidazole (4a)}

White solid, yield: $76 \%$; mp: $168-170{ }^{\circ} \mathrm{C} ;{ }^{1} \mathrm{H}$ NMR (300 MHz, DMSO-d 6 ): $\delta 4.80$ (s, 2H, $\mathrm{CH}_{2}$-S-), 7.10-8.00 (m, 9H, Ar-H), 12.60 (s, 1H, $\mathrm{D}_{2} \mathrm{O}$ exch., $\left.-\mathrm{NH}\right)$.

\section{2-((5-(4-Methoxyphenyl)-1,3,4-oxadiazol-2-ylthio)methyl)-1H-benzimidazole (4b)}

Light yellow crystal, yield: $53.9 \%$, mp: $120-121{ }^{\circ} \mathrm{C} .{ }^{1} \mathrm{H}$ NMR $\left(300 \mathrm{MHz}, \mathrm{DMSO}-d_{6}\right) \delta$ : $3.85(\mathrm{~s}, 3 \mathrm{H}), 4.58(\mathrm{~s}, 2 \mathrm{H}), 7.13(\mathrm{~d}, J=8.8 \mathrm{~Hz}, 2 \mathrm{H}), 7.51(\mathrm{~d}, J=8.2 \mathrm{~Hz}, 1 \mathrm{H}), 7.88(\mathrm{~d}$, $J=8.8 \mathrm{~Hz}, 2 \mathrm{H}), 7.98(\mathrm{~d}, J=8.2 \mathrm{~Hz}, 1 \mathrm{H}), 8.50(\mathrm{~s}, 1 \mathrm{H})$. 
2-((5-(4-Nitrophenyl)-1,3,4-oxadiazol-2-yl)thiomethyl)-1H-benzimidazole (4c)

White solid, yield: $76 \%$; mp: $168-170{ }^{\circ} \mathrm{C} ;{ }^{1} \mathrm{H}$ NMR (300 MHz, DMSO-d 6 ): $\delta 4.90$ (s, 2H, $\mathrm{CH}_{2}-\mathrm{S}-$ ), 7.30-8.40 ( $\left.\mathrm{m}, 8 \mathrm{H}, \mathrm{Ar}-\mathrm{H}\right), 12.92$ (s, 1H, $\mathrm{D}_{2} \mathrm{O}$ exch., $-\mathrm{NH}$ ).

2-((5-(4-Methyphenyl)l-1,3,4-oxadiazol-2-yl)thiomethyl)-1H-benzimidazole(4d)

White solid, yield: $76 \%$; mp: $202-205{ }^{\circ} \mathrm{C}$; IR (KBr): ${ }^{1} \mathrm{H}-\mathrm{NMR}\left(300 \mathrm{MHz}, \mathrm{DMSO}-\mathrm{d}_{6}\right): \delta 2.30$ (s, $3 \mathrm{H}, \mathrm{Ar}-\mathrm{CH}_{3}$ ), 4.80 (s, 2H, $\left.-\mathrm{CH}_{2}-\mathrm{S}-\right), 7.00-8.00$ (m, 8H, $\left.\mathrm{Ar}-\mathrm{H}\right), 12.50\left(\mathrm{~s}, 1 \mathrm{H}, \mathrm{D}_{2} \mathrm{O}\right.$ exch., $\left.-\mathrm{NH}\right)$.

2-((5-(4-Tert-Butoxyphenyl)-1,3,4-oxadiazol-2-ylthio)methyl)-1H-benzimidzole(4e)

White solid, yield: 51.4\%; mp: $195-197{ }^{\circ} \mathrm{C}$; ${ }^{1} \mathrm{H}$ NMR $\left(300 \mathrm{MHz}, \mathrm{DMSO}-\mathrm{d}_{6}\right) \delta 1.24$ (s, 9H), $7.36\left(\mathrm{~s}, 2 \mathrm{H}, \mathrm{NH}_{2}\right), 7.61-7.81(\mathrm{~m}, 9 \mathrm{H}, \mathrm{Ar}-\mathrm{H})$

2-((5-(4-Fluorophenyl)-1,3,4-oxadiazol-2-ylthio)methyl)-1H-benzoimidazole(4f)

Light yellow powder, yield: $56.2 \%$, mp: $132-133{ }^{\circ} \mathrm{C} .{ }^{1} \mathrm{H}$ NMR $\left(300 \mathrm{MHz}, \mathrm{DMSO}-d_{6}\right) \delta$ : $4.61(\mathrm{~s}, 2 \mathrm{H}), 7.45-8.04(\mathrm{~m}, 3 \mathrm{H}), 8.53(\mathrm{~s}, 1 \mathrm{H})$.

4-(5-((1H-Benzimidazol-2-yl)methylthio)-1,3,4-oxadiazol-2-yl)-benzenamine(4g)

White solid, yield: $76 \%$; mp: $168-170{ }^{\circ} \mathrm{C} ;{ }^{1} \mathrm{H}$ NMR (300 MHz, DMSO-d 6 ): $\delta: 4.90$ (s, 2H, $\mathrm{CH}_{2}$-S-), 5.97 (s, 2H, -NH $\mathrm{NH}_{2}$, 7.30-8.40 ( m, 8H, Ar-H), 12.92 (s, 1H, $\mathrm{D}_{2} \mathrm{O}$ exch., -NH).

2-((5-(4-Chlorophenyl)-1,3,4-oxadiazol-2-ylthio)methyl)-1H-benzimidazole(4h)

White solid, yield: $76 \%$; mp: $145-147{ }^{\circ} \mathrm{C} ;{ }^{1} \mathrm{H}$ NMR (300 MHz, DMSO-d 6 ): $\delta 4.68$ (s, 2H, $\mathrm{CH}_{2}$-S-), 7.20-7.90 (m, 8H, Ar-H), 12.60 (s, 1H, $\mathrm{D}_{2} \mathrm{O}$ exch., $\mathrm{NH}$ ).

2-((5-(4-Bromophenyl)-1,3,4-oxadiazol-2-ylthio)methyl)-1H-benzimidazole(4i)

White powder, yield: $76 \%$; mp: $145-147{ }^{\circ} \mathrm{C} ;{ }^{1} \mathrm{H}$ NMR (300 MHz, DMSO-d 6 ): $\delta 4.68$ (s, 2H, $-\mathrm{CH}_{2}$-S-), 7.20-7.90 (m, 8H, Ar-H), 12.60 (s, 1H, D $\mathrm{D}_{2} \mathrm{O}$ exch., NH).

\section{General procedure for compounds $\mathbf{5 a - i}$}

To a solution of compound $4(0.01 \mathrm{~mol})$ in dimethylformamide $(25 \mathrm{~mL})$, sodium hydride $(0.0 \mathrm{~mol})$ was added gradually under ice cooling. The reaction mixture was stirred for 30 min and then the appropriate halide $(0.01 \mathrm{~mol})$ was added portion wise. The whole mixture was warmed to room temperature and stirred for $4 \mathrm{~h}$, poured onto ice-water with continuous stirring. The precipitated product was filtered off and purified by silica gel columnchromatography.

\section{2-((5-Phenyl-1,3,4-oxadiazol-2-ylthio)methyl)-1-benzylbenzimidazole (5a)}

White solid,yield:76\%; mp: $168-170{ }^{\circ} \mathrm{C} ;{ }^{1} \mathrm{H}$ NMR (300 MHz, DMSO-d 6 ): $\delta 4.80$ (s, 2H, $\left.\mathrm{CH}_{2}-\mathrm{S}-\right), 5.13\left(\mathrm{~s}, 2 \mathrm{H}, \mathrm{CH}_{2}\right), 7.06-7.88(\mathrm{~m}, 14 \mathrm{H}, \mathrm{Ar}-\mathrm{H}) ;{ }^{13} \mathrm{C}-\mathrm{NMR}\left(\mathrm{DMSO} \mathrm{d}_{6}\right) \delta 20.3\left(-\mathrm{CH}_{2}-\right.$ S-), 122.1, 122.9, 126.4, 129.3, 132.0, 138.1, 152.9, 161.5, 165.6 (Ar-H); Anal. Calcd. For $\mathrm{C}_{23} \mathrm{H}_{18} \mathrm{~N}_{4} \mathrm{OS}$ : C, 69.32; H, 4.55; N, 14.06. Found:C, 69.30; H, 4.92; N, 14.19.

2-((5-(4-Methoxyphenyl)-1,3,4-oxadiazol-2-yl]thiomethyl\}-1-benzylbenzimidazole (5b)

Pale brown solid, yield: 66\%; mp: $168-170{ }^{\circ} \mathrm{C}$; ${ }^{1} \mathrm{H}$ NMR (300 MHz, DMSO-d 6 ): $\delta 3.72\left(\mathrm{~s}, 3 \mathrm{H},-\mathrm{OCH}_{3}\right), 4.24\left(\mathrm{~s}, 2 \mathrm{H},-\mathrm{CH}_{2}-\mathrm{S}-\right), 5.04\left(\mathrm{~s}, 2 \mathrm{H}, \mathrm{CH}_{2}\right), 7.10-8.00(\mathrm{~m}, \mathrm{Ar}-\mathrm{H})$; Anal. Calcd. For $\mathrm{C}_{24} \mathrm{H}_{20} \mathrm{~N}_{4} \mathrm{O}_{2} \mathrm{~S}: \mathrm{C}, 67.27 ; \mathrm{H}, 4.70, \mathrm{~N}, 13.07$. Found:C, 67.30; H, 4.92; N, 13.19 . 
2-((5-(4-Nitrophenyl)-1,3,4-oxadiazol-2-yl)thiomethyl)-1-benzylbenzimidazole (5c) Yellow solid, yield: 52\%; mp: $168-170{ }^{\circ} \mathrm{C} ;{ }^{1} \mathrm{H}$ NMR (300 MHz, DMSO-d $\left.{ }_{6}\right): \delta 4.20$ (s, 2H, $\left.\mathrm{CH}_{2}-\mathrm{S}-\right)$, 5.54 (s, 2H, $\mathrm{CH}_{2}$ ), 7.30-8.40 ( m, 13H, Ar-H);. Anal.Calcd.for $\mathrm{C}_{23} \mathrm{H}_{17} \mathrm{~N}_{5} \mathrm{O}_{3} \mathrm{~S}: \mathrm{C}$, 62.29; H, 3.86; N, 15.79. Found:C, 62.36; H, 3.15; N, 15.80 .

\section{2-((5-(4-Methylphenyl)-1,3,4-oxadiazol-2-yl)thiomethyl\}-1-benzylbenzimidazole (5d)}

White solid, yield: $68 \%$; mp: 202-205 ${ }^{\circ} \mathrm{C}$; ${ }^{1} \mathrm{H}$ NMR (300 MHz, DMSO-d 6 ): $\delta 2.32$ (s, 3H, Ar- $\left.\mathrm{CH}_{3}\right), 4.28$ (s, 2H, - $\left.\mathrm{CH}_{2}-\mathrm{S}-\right), 5.04\left(\mathrm{~s}, 2 \mathrm{H}, \mathrm{CH}_{2}\right), 7.00-7.98$ (m,Ar-H); Anal. Calcd.for $\mathrm{C}_{24} \mathrm{H}_{20} \mathrm{~N}_{4} \mathrm{OS}$ : C, 69.88; H, 4.89; N, 13.58. Found: C, 69.30; H, 4.86; N, 13.37 .

\section{2-((5-(4-Tert-butylphenyl)-1,3,4-oxadiazol-2-yl)thiomethyl)-1-benzylbenzimidazole (5e)}

White solid, yield: 56\%; mp: 202-205 ${ }^{\circ} \mathrm{C} ;{ }^{1} \mathrm{H}$ NMR (300 MHz, DMSO-d 6 ): $\delta 1.32$ (s, 9H, $\mathrm{C}\left(\mathrm{CH}_{3}\right)_{3}, 4.20$ (s, 2H, - $\left.\mathrm{CH}_{2}-\mathrm{S}-\right), 5.02$ (s, 2H, $\left.\mathrm{CH}_{2}\right), 6.98$ - 7.78 (m, Ar-H); Anal. Calcd.for $\mathrm{C}_{27} \mathrm{H}_{26} \mathrm{~N}_{4} \mathrm{OS}$ : C, 71.34; H, 5.76; N, 12.32. Found:C, 71.55; H, 5.02; N, 12.46.

2-((5-(4-Fluorophenyl)-1,3,4-oxadiazol-2-yl)thiomethyl)-1-benzylbenzimidazole (5f)

Light yellow crystal, yield: $48 \%$, mp: $132-133{ }^{\circ} \mathrm{C} .{ }^{1} \mathrm{H}$ NMR $\left(300 \mathrm{MHz}, \mathrm{DMSO}-d_{6}\right) \delta: 4.54$ (s, 2H, - $\mathrm{CH}_{2}-\mathrm{S}-$ ), 4.61 (s, 2H), 6.85-7.53 (m, Ar-H), Anal.Calcd.for $\mathrm{C}_{23} \mathrm{H}_{17} \mathrm{FN}_{4} \mathrm{OS}: \mathrm{C}, 66.33$; H, 4.11; N, 13.45. Found: C, 66.38; H, 4.77; N, 13. 01.

\section{4-(5-((-Benzylbenzimidazol-2-yl)methylthio)-1,3,4-oxadiazol-2-yl)benzenamine (5g)}

Yellow solid, yield: $76 \%$; mp: $202-205{ }^{\circ} \mathrm{C} ;{ }^{1} \mathrm{H}$ NMR (300 MHz, DMSO-d ${ }_{6}$ ): $\delta 3.80$ (s, 2H, $\left.\mathrm{NH}_{2}\right), 4.20$ (s, 2H, $\left.-\mathrm{CH}_{2}-\mathrm{S}-\right), 5.02\left(\mathrm{~s}, 2 \mathrm{H}, \mathrm{CH}_{2}\right), 6.58-7.50$ (m, 13H, Ar-H); Anal. Calcd. for $\mathrm{C}_{23} \mathrm{H}_{19} \mathrm{~N}_{5} \mathrm{OS}$ : C, 66.81; H, 4.63; N, 16.94. Found: C, 66.55; H, 4.02; N, 16.46.

2-((5-(4-Chlorophenyl)-1,3,4-oxadiazol-2-yl)thiomethyl)-1-benzylbenzimidazole (5h)

Light yellow crystal, yield: $56.2 \%$, mp: $152-155^{\circ} \mathrm{C} .{ }^{1} \mathrm{H}$ NMR $\left(300 \mathrm{MHz}, \mathrm{DMSO}-d_{6}\right) \delta: 4.54$ (s, 2H, - $\mathrm{CH}_{2}-\mathrm{S}-$ ), 4.61 (s, 2H), 6.36-7.53 (m, 13H,Ar-H), Anal. Calcd.for $\mathrm{C}_{23} \mathrm{H}_{17} \mathrm{ClN}_{4} \mathrm{OS}: \mathrm{C}$, 66.33; H, 4.11; N, 13.45. Found: C, 66.38; H, 4.77; N, 13. 01.

\section{2-((5-(4-Bromophenyl)-1,3,4-oxadiazol-2-yl)thiomethyl)-1-benzylbenzimidazole (5i)}

Light yellow crystal, yield: $56.2 \%$, mp: $122-125{ }^{\circ} \mathrm{C} .{ }^{1} \mathrm{H}$ NMR $\left(300 \mathrm{MHz}, \mathrm{DMSO}-d_{6}\right) \delta: 4.58$ (s, 2H, - $\left.\mathrm{CH}_{2}-\mathrm{S}-\right), 4.57$ (s, 2H), 6.85-7.53 (m,13H,Ar-H), Anal. Calcd. for $\mathrm{C}_{23} \mathrm{H}_{17} \mathrm{BrN}_{4} \mathrm{OS}: \mathrm{C}$, 57.87; H, 3.59; N, 11.74. Found: C, 57.38; H, 3.59; N, 11. 01.

\section{In vitro anticancer screening}

Hanks balanced salt solution, (4-(2-hydroxyethyl)-1-piperazineethanesulfonic acid) (HEPES), ethanol, 96 well plates, general reagents and supplies, were all purchased from Sigma-Aldrich Co. (St. Louis, MO) and VWR International (Radnor, PA). Imaging probes were supplied by Life Technologies Grand Island, NY.

\section{Cell culture}

MDA-MB-231 (ATCC $\left({ }^{\circ}\right.$ HTB-26 ${ }^{\text {TM }}$ ) human breast cancer cells were obtained from ATCC (Manassas, VA). MDA-MB-231 cells were brought up in ATCC-formulated Leibovitz's L-15 Medium [Catalog No. 30-2008], supplemented with 10\% FBS and penicillin/streptomycin $(100 \mathrm{U} / 0.1 \mathrm{mg} / \mathrm{mL})$. After confluence, the cells were sub-cultured 
and grown in DMEM containing phenol red, 10\% FBS, $4 \mathrm{mM}$ L-glutamine, $20 \mu \mathrm{M}$ sodium pyruvate and penicillin/streptomycin $(100 \mathrm{U} / 0.1 \mathrm{mg} / \mathrm{mL})$. Culture conditions were maintained $\left[37{ }^{\circ} \mathrm{C}\right.$ in $5 \% \mathrm{CO}_{2} /$ atmosphere] and every $2-5$ days, the media was replaced and cells sub-cultured.

\section{Proliferation and toxicity screening}

Compounds were dissolved in DMSO, vortexed and stored at $-20{ }^{\circ} \mathrm{C}$ in the dark. A stock solution for each experimental compound was prepared in $\mathrm{HBSS}+5 \mathrm{mM}(N$-[2hydroxyethyl-piperazine]- $N^{\prime}$-[2-ethanesulfonic acid]) (HEPES), adjusted to a $\mathrm{pH}$ of 7.4. An initial screening was conducted at 5 concentrations within the test range of 0.416 $\mathrm{mg} / \mathrm{mL}$ to $0.0208 \mathrm{mg} / \mathrm{mL}$ for toxicity and mitostatic potential. Briefly, for cell proliferation studies, 96 well plates contained a low cell plating density $\left[0.04 \times 10^{6} /\right.$ well $]$ and full growth media \pm experimental compounds. A 72 hour cell count was evaluated relative to positive and negative (paclitaxel) controls. The toxic effects of each compound were evaluated at 24 hours, where cells were plated in low serum media at higher plating cell plating density $\left[0.4 \times 10^{6} /\right.$ well $] \pm$ experimental compounds with positive and negative (paclitaxel) controls.

\section{Results and Discussion}

\section{Chemistry}

The synthetic strategies adopted for the synthesis of the target compounds are depicted in Scheme 1. Condensation of $\mathbf{3}$ with 2-chloromethylbenzimidazole in presence of potassium hydroxide in ethanol afforded compound 4. The reaction was comprised of two steps; the first step is the formation of a salt and in the second step the salt was treated with 2chloromethyl- benzimidazole afforded $\mathbf{4}$ in fair to good yields. $N$-benzylation of $\mathbf{4}$ with benzyl bromide in presence of sodium hydride in DMF afforded $\mathbf{5}$ in fair to good yields.

The structures of the newly synthesized compounds were established on the basis IR, ${ }^{1} \mathrm{H}$ $\mathrm{NMR}$ and ${ }^{13} \mathrm{C}$ NMR spectrum. In general, IR spectra of the compounds showed peaks at 3326 and $2557 \mathrm{~cm}^{-1}$ for $\mathrm{NH}$ and $\mathrm{SH}$, respectively. In ${ }^{1} \mathrm{H}$ NMR spectra, there appeared a singlet at around $\delta 11.9$ indicative of ring $\mathrm{H}-\mathrm{N}$ and another singlet at $\delta 13.4$ for $\mathrm{S}-\mathrm{H}$, both disappeared by addition of $\mathrm{D}_{2} \mathrm{O}$ as confirmation for these groups. The ${ }^{1} \mathrm{H}$ NMR of 4 showed disappearance of broad singlet of SH and corresponding appearance of the singlet at $\delta 4.35$ $4.15 \mathrm{ppm}$ integrating for two protons due to $\mathrm{S}-\mathrm{CH}_{2}$ groupforming a linker through which benzimidazole nuclei attached with oxadiazole nuclei. The chemical shift in ${ }^{13} \mathrm{C} \mathrm{NMR}$ spectra at $\delta 173.56$ and 156.24 could be accounted for $\mathrm{C}=\mathrm{O}$ and $\mathrm{C}=\mathrm{N}$. All target compounds showed a singlet at chemical shift around $\delta 5.0$ in ${ }^{1} \mathrm{H}$ NMR spectra and ${ }^{13} \mathrm{C}$ NMR spectra showed at around $\delta 30.9$ which could be accounted for $\mathrm{N}_{-} \mathrm{CH}_{2}$ group. The multiplet peaks shown at around $\delta$ 7.2-6.9 accounted for aromatic protons.

\section{Anticancer assay}

The synthesized compounds were screened for their in vitro anticancer potential against a panel of breast cancer cell lines: MDA-MB-231 using paclitaxel as control. The relationship between surviving fraction of cells and drug concentration was plotted and the response parameter $\mathrm{IC}_{50}$ was calculated. $\mathrm{IC}_{50}$ value corresponds to the concentration required for $50 \%$ inhibition of cell viability. The $\mathrm{IC}_{50}$ value of the test compounds are shown in Table 1. Compound 5c exhibited moderate inhibition potency $(<50 \mu \mathrm{M})$ as described in Table 1. 
Table 1. $\mathrm{IC}_{50}$ value of the test compounds<smiles>COc1ccc(-c2nnc(SCc3nc4ccccc4n3Cc3ccccc3)o2)cc1</smiles>

\section{Conclusion}

The present work, through simple synthetic approaches, led to the development of a hybrid of benzimidazoles containing 1,3,4-oxadiazolepharmacophore that exhibited moderate cytotoxic activities against breast tumor cell lines. The activity could be attributed to some sort of synergism between benzimidazole coupled with 1,3,4-oxadiazole molecule. 


\section{Acknowledgment}

The authors are grateful to the National Institute of Health, the National Institute of General Medical Sciences, MBRS Program (GM 08111) and Research Centre at Minority Institutions Grant (RCMI) RR 03020. And School of Graduate School, Faculty Research Award.

\section{References}

1. YogitaBansal and Om Silakari, Bioorg Med Chem., 2012, 20(21), 6208-6236; DOI:10.1016/j.bmc.2012.09.013

2. Ansari K F and Lal C, Eur J Med Chem., 2009, 44(10), 4028-4033; DOI:10.1016/j.ejmech.2009.04.037

3. Heba T Abdel-Mohsen, Fatma A F Ragab, Mostafa M Ramla and Hoda I El Diwani, Eur J Med Chem., 2010, 45(6), 2336-2344; DOI:10.1016/j.ejmech.2010.02.011

4. Asif Husain, Mohd Rashid, M. Shaharyar, Anees A. Siddiqui, Ravinesh Mishra, Eur J Med Chem., 2013, 62, 785-798; DOI:10.1016/j.ejmech.2012.07.011

5. Pace A and Pierro P, Org Biomol Chem., 2009; 7, 4337-4348; DOI:10.1039/B908937C

6. De Oliveira C S, Lira B F, Barbosa-Filho J M, Lorenzo J G, deAthavde-Filho P F. Molecules, 2012; 17(9), 10192.

7. Sun J, Makawana J A and Zhu H L, Mini Rev Med Chem., 2013, 13(12), 1725-1743.

8. Basoglu S, Yolal M, Demirci S, Demirbas N, Bektas H and Karaoglu S A, Acta Pol Pharm., 2013; 70(2), 229-236.

9. Saha R. Tanwar, Mareella A, Alam M.M and Akhter M, Mini Rev Med Chem., 2013, 13, 1027-1046. 